OPEN ACCESS

Edited by:

Claudia Penna

University of Turin, Italy

Reviewed by:

Barbara Ruszkowska-Ciastek,

Nicolaus Copernicus University

in Toruń, Poland

Claudio Molinari,

University of Eastern Piedmont, Italy

*Correspondence:

Jie L

lijie860114@163.com

Chun Wang

dysrmyysjnkwc@163.com

${ }^{\dagger}$ These authors have contributed equally to this work

Specialty section:

This article was submitted to

Vascular Physiology,

a section of the journa

Frontiers in Physiology

Received: 15 December 2020

Accepted: 18 March 2021

Published: 09 April 2021

Citation:

Li J, Gao L, Zhang P, Liu Y,

Zhou J, Yi X and Wang C (2021)

Vulnerable Plaque Is More Prevalent

in Male Individuals at High Risk

of Stroke: A Propensity

Score-Matched Study

Front. Physiol. 12:642192.

doi: 10.3389/fphys.2021.642192

\section{Vulnerable Plaque Is More Prevalent in Male Individuals at High Risk of Stroke: A Propensity Score-Matched Study}

\author{
Jie Li ${ }^{1 *}$, Lijie Gao ${ }^{2 \dagger}$, Ping Zhang ${ }^{1}$, Yingying Liu' ${ }^{1}$, Ju Zhou' ${ }^{1}$, Xingyang Yi' and \\ Chun Wang ${ }^{1 *}$ \\ ${ }^{1}$ Department of Neurology, People's Hospital of Deyang City, Deyang, China, ${ }^{2}$ Department of Neurology, West China \\ Hospital, Sichuan University, Chengdu, China
}

Objectives: To assess the gender differences in the prevalence of carotid vulnerable plaques in high-risk individuals for stroke in a multicenter, cross-sectional study.

Methods: In the year 2015, 18595 residents who were at the age of 40 or older participated in a face-to-face study in eight communities in southwestern China. Totally 2,644 participants at high risk of stroke were enrolled. Before and after propensity score matching (PSM), the prevalence of carotid plaques and vulnerable plaques were compared between men and women. Multivariate analyses were applied to explore the association between the gender and carotid plaques. Stratified analyses and interaction tests were performed to identify factors that might modify the association between the gender and carotid plaques.

Results: Among 2644 high-risk individuals enrolled, there were 1,202 (45.5\%) men and 1442 (54.5\%) women. Carotid plaques were detected in 904 (34.2\%) participants, while vulnerable plaques were found in 425 (16.1\%) participants. Before PSM, carotid plaques were more prevalent in male individuals than the female (36.7\% vs. 32.1\%, $p=0.01)$, as well as vulnerable plaque $(20.0 \%$ vs. $12.8 \%, p<0.01)$. Men tend to have a higher prevalence of vulnerable plaques in multivariate analyses (adjusted OR $1.70,95 \% \mathrm{Cl}$ 1.10-2.62, $p=0.02$ ). Stratified analyses and interaction tests demonstrated that the association between male sex and vulnerable carotid plaque did not change by age, family history of stroke, histories of chronic disease, smoking status, drinking status, physical activity, and BMI (all $p$ for interaction > 0.05). After PSM, vulnerable plaques were still more prevalent in male individuals than the female $(17.03 \%$ vs. $12.07 \%$, $p=0.032)$.

Conclusion: Male individuals had a higher risk of vulnerable carotid plaque independent of classical vascular risk factors. Whether there is a gender-specific association between variations in genes related to inflammation, lipid metabolis, and endothelial function and plaque vulnerability needs to be further studied.

Keywords: gender, plaque vulnerability, risk factors, propensity score matching, atherosclerosis 


\section{INTRODUCTION}

Stroke is one of the leading causes of death and the major cause of adult disability worldwide, especially in China (GBD 2016 Causes of Death Collaborators, 2017; Wu et al., 2019). With the aging of the population, the onging high incidence of risk factors and inadequate management, the burden of stroke is increasing year by year (Wu et al., 2019). Approximately $80 \%$ of all strokes are ischemic and carotid artery atherosclerosis accounts for at least 20\% of all ischemic strokes (Prasad, 2015; Puig et al., 2020).

Atherosclerosis is an chronic inflammatory disease of the arterial wall, with the characteristics of inflammation, endothelial injury, lipid accumulation, and extensive degradation of extracellular matrix components (Mangge and Almer, 2019; Wijeratne et al., 2020). Carotid atherosclerosis has been identified as a major risk factor of ischemic stroke, cardiovascular diseases, and other vascular events (Rundek et al., 2008; Sillesen et al., 2018; Parish et al., 2019). Ultrasound is a non-invasive and economical diagnostic technique that helps provide valuable information on carotid atherosclerosis such as carotid intima thickness (CIMT) and carotid plaque presence (Park, 2016). Several studies suggest that carotid plaque is more powerful in predicting vascular outcomes, compared with CIMT (Ho, 2016; Nezu and Hosomi, 2020).

Previous epidemiological researches have reported the associations between several classical vascular risk factors (such as age, hypertension, diabetes, dyslipidemia, and current smoking) and carotid plaques (Sturlaugsdottir et al., 2016; Bian et al., 2018; Noflatscher et al., 2019; Santos-Neto et al., 2021). It is noted that the incidence of stroke is higher in male individuals compared with the female age $<75$ years (Lloyd-Jones et al., 2010), and gender differences in plaque characteristics might help explain this phenomenon. However, there is scarce information available about the gender differences in the prevalence of carotid plaque in high-risk individuals for stroke. Previous studies which investigated the association between sex and intra-plaque hemorrhage (IPH) mainly focused on patients with moderate or severe carotid stenosis (Ota et al., 2010). Meanwhile, the judgment of IPH in carotid plaque was mainly based on histopathological examination after carotid endarterectomy (CEA) (Hellings et al., 2007). Therefore, we conducted the present study using the data of a multicenter, cross-sectional survey in China to explore the gender differences in the prevalence of carotid plaques among individuals at high risk of stroke.

\section{MATERIALS AND METHODS}

\section{Study Design and Participants}

The present study was a branch of the China National Stroke Screening Survey (CNSSS) program of the National Health and Family Planning Commission of China (grant No. 2011BAI08B01) (Li et al., 2015; Yi et al., 2020a,b). The CNSSS which aimed to provide stroke prevention policies for the Chinese, is a population-based cross-sectional study with a 2-stage stratified sampling framework (Li et al., 2015;
Yi et al., 2020a,b). More details of the CNSSS could be followed at the official website (Stroke Prevention Project Committee, 2018). From May 1, 2015 to Sep 31, 2015, the present study was conducted in eight randomly selected communities of Sichuan province in southwestern China, using a cluster survey method (Yi et al., 2020a,b). This survey was performed among residents aged $\geq 40$ and who lived more than 6 -month in each community. Ethics Committee of the three participating institutions (People's Hospital of Deyang City, Affiliated Hospital of Southwest Medical University, the Suining Central Hospital) approved our study protocol and written informed consent was obtained from all participants enrolled in this study (Yi et al., 2020a,b).

\section{Data Collection and the Definition of High-Risk Individuals for Stroke}

Data were collected via using a standardized structured faceto-face questionnaire by experienced surveyors, including demographic information (age, sex, education level), family history of stroke, behavior factors (smoking, drinking, exercise habits), history of stroke [ischemic stroke or transient ischemic attack (TIA), hemorrhagic stroke], history of chronic diseases (hypertension, dyslipidemia, diabetes mellitus, and atrial fibrillation) (Yi et al., 2020a,b). Body measurements of height, weight, waist circumference, and hip circumference were also measured and recorded in the questionnaire. The eight stroke-related risk factors were assessed, including hypertension, dyslipidemia, diabetes mellitus, atrial fibrillation, current smoking ( $\geq 1$ cigarette per day), physical inactivity (physical exercise $<3$ times per week for $<30$ min each time), overweight/obesity [defined as body mass index $\left.(B M I) \geq 26 \mathrm{~kg} / \mathrm{m}^{2}\right]$, and a family history of stroke, which has been elaborated upon in our previous study (Yi et al., 2020a,b). Participants who had at least three of the above eight risk factors or had a history of stroke were identified as the high-risk participants for stroke (Wang et al., 2017).

Laboratory examinations such as fasting blood glucose (FBG), hemoglobin A1c, triglycerides, total cholesterol (TC), highdensity lipoprotein cholesterol (HDL-C), low-density lipoprotein cholesterol (LDL-C), and homocysteine, electrocardiogram (ECG), and carotid ultrasonography were also obtained from the high-risk participants for stroke (Yi et al., 2020a,b). Detailed methods for data collection have been elaborated upon in our previous studies (Yi et al., 2020a,b).

\section{Carotid Ultrasound Examination}

Diagnostic ultrasound (type 512, ACUSON Sequoia Apparatus, $7.5 \mathrm{MHz}$ probe, Berlin, Germany) was performed in participants at high risk of stroke to assessed bilateral common and internal carotid arteries, as well as bifurcations according to standard scanning reading protocols (Rundek et al., 2008; Yi et al., 2020b). Detailed procedures for evaluating the characteristics of carotid plaque have been described in our previous study (Yi et al., 2016, 2017, 2020b). An atherosclerotic plaque was defined as the presence of an endoluminal protrusion $>1.5 \mathrm{~mm}$ or a focal thickening at least $50 \%$ greater of the CIMT than adjacent arterial wall (Rundek et al., 2008; Yi et al., 2020b). 
Based on the plaque echogenicity and surface appearance, carotid plaques were further classified from class I to class IV as uniformly echolucent, predominantly echolucent, predominantly echogenic, and echogenic, respectively (Mathiesen et al., 2001; Yi et al., 2020b). Plaques of class I or II were identified as vulnerable plaques, while plaques of class III or IV were identified as stable plaques (Yi et al., 2016, 2017, 2020b). Carotid plaques were independently classified by ultrasound practitioners who were blinded to baseline information.

\section{Statistical Analyses}

Clinical characteristics are presented as means with standard deviations (SDs) for continuous variables and as frequencies with percentages for categorical variables according to different genders. Intergroup differences in categorical variables were calculated for significance using the $\chi^{2}$-tests or Fisher's exact tests, while intergroup differences in continuous variables were calculated using the Student's $t$-tests or Mann-Whitney $U$-test (Li et al., 2020a).

Univariate analysis comparing factors associated with carotid plaque and vulnerable plaque in high-risk individuals for stroke was performed. Multivariate logistic regression was performed to identify the association between gender and carotid plaque in high-risk individuals in 4 different models. Model 1 was adjusted for age and family history of stroke. Model 2 was adjusted for variables in model $1+$ BMI. Model 3 was adjusted for variables in model $1+\mathrm{BMI}+$ vascular risk factors (history of ischemic stroke or TIA, hypertension, dyslipidemia, diabetes mellitus, smoking status). Model 4 was adjusted for variables in model $1+$ BMI + vascular risk factors + laboratory test (Hemoglobin A1c, FBG, Triglycerides, TC, HDL-C, LDL-C). Stratified analyses and interaction tests were conducted according to age, family history of stroke, histories of chronic disease, smoking status, drinking status, physical activity, and BMI, to identify factors that might modify the association between the gender and carotid plaques. The significance of interaction was tested by the loglikelihood ratio test.

We also performed a propensity score matching (PSM) algorithm including baseline characteristics that are assumed to be related to the gender by using a multivariate logistic regression analysis, to calculate the propensity score for each patient. Then participants between different gender groups were matched via using the nearest neighbor approach (caliper 0.2 , ratio 1:1) to minimize potential imbalances between the two groups as previously described in detail (Li et al., 2020a). Gender differences in the prevalence of carotid plaques and vulnerable plaques were compared before and after PSM.

The 95\% confidence intervals (CI) were calculated to describe the precision of the estimates. Two-sided $P<0.05$ was considered statistically significant for all results. All statistical analyses were performed using SPSS 21.0 software (IBM, Chicago, IL, United States), statistical software packages R (The R Foundation, version 3.4.3) ${ }^{1}$ and EmpowerStats (X\&Y Solutions, Inc., Boston,

${ }^{1}$ http://www.R-project.org
MA, United States) ${ }^{2}$, which have been described in our previous studies (Li et al., 2020a,b).

\section{RESULTS}

In the year 2015,18595 residents aged $\geq 40$ participated in the face-to-face survey in eight communities in Sichuan province in southwestern China. Finally, a total of 2644 subjects at high risk of stroke were enrolled, comprising 1,202 men and 1,442 women aged $63.3 \pm 9.8$ years. A flow diagram of the data preparing and cleaning process in this survey is provided in Figure $\mathbf{1}$.

\section{Gender Differences in the Characteristics of High-Risk Individuals and the Prevalence of Carotid Plaque}

Gender differences in the characteristics of high-risk individuals and the prevalence of carotid plaque were exhibited in Table 1. Compared with women, men were younger $(62.7 \pm 10.3$ vs. $63.7 \pm 9.4$ years, $p<0.01)$, had higher educational level $(p<0.01)$, more history of former and current smoking (13.9\%, $54.2 \%$ vs. $1.7 \%, 4.4 \%$, respectively, $p<0.01$ ) and regular alcohol consumption $(18.7 \%$ vs. $1.6 \%, p<0.01)$, less history of ischemic stroke or transient ischemic stroke (TIA) $(14.4 \%$ vs. $20.5 \%, p<0.01)$, diabetes $(28.4 \%$ vs. $39.3 \%$, $p<0.01)$, dyslipidemia $(67.8 \%$ vs. $76.5 \%, p<0.01)$ and atrial fibrillation $(7.8 \%$ vs. $11.0 \%, p<0.01)$. Meanwhile, the level of FBG $(6.3 \pm 2.2$ vs. $6.5 \pm 2.7 \mathrm{mmol} / \mathrm{L}, p=0.02)$, total cholesterol $(5.1 \pm 1.0$ vs. $5.3 \pm 1.0 \mathrm{mmol} / \mathrm{L}, p<0.01)$, HDL-C (1.5 \pm 0.5 vs. $1.7 \pm 0.6 \mathrm{mmol} / \mathrm{L}, p<0.01)$ and LDL-C $(2.9 \pm 0.9$ vs. $3.1 \pm 0.9 \mathrm{mmol} / \mathrm{L}, p<0.01)$ were lower in men than in women in the current survey. However, men had a higher level of homocysteine than women (15.5 \pm 11.1 vs. $11.9 \pm 7.2 \mathrm{mmol} / \mathrm{L}, p<0.01)$.

${ }^{2}$ http://www.empowerstats.com

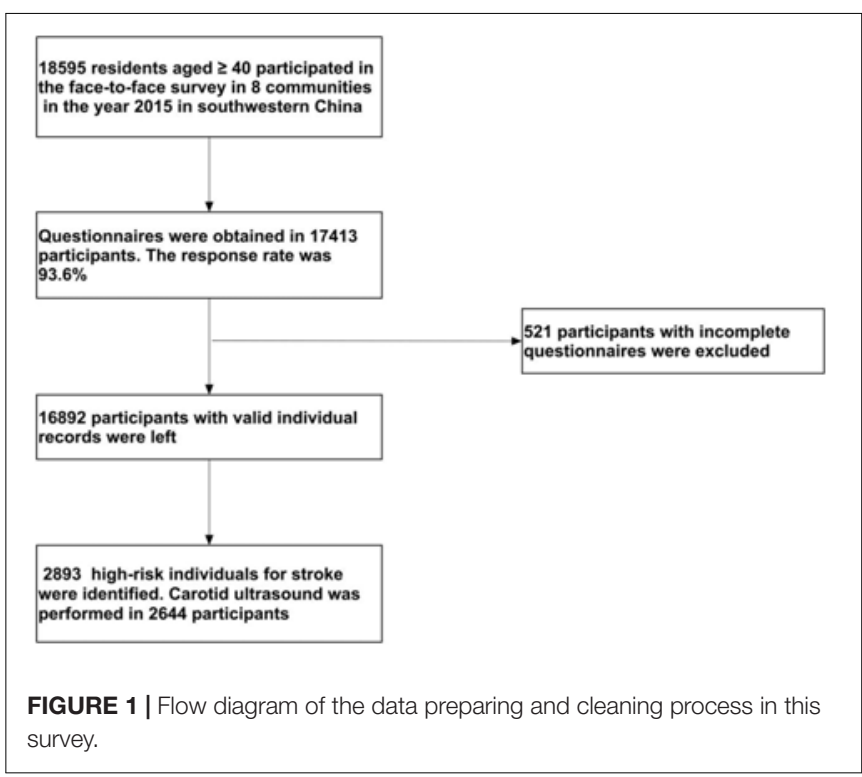


TABLE 1 | Gender differences in the characteristics of individuals at high risk of stroke and the prevalence of carotid plaque.

\begin{tabular}{|c|c|c|c|c|}
\hline Variables & $\begin{array}{c}\text { Total } \\
(n=2,644)\end{array}$ & $\begin{array}{c}\text { Male } \\
(n=1,202)\end{array}$ & $\begin{array}{c}\text { Female } \\
(n=1,442)\end{array}$ & $P$-value \\
\hline Age, year, mean $\pm S D$ & $63.3 \pm 9.8$ & $62.7 \pm 10.3$ & $63.7 \pm 9.4$ & $<0.01^{*}$ \\
\hline Education, n (\%) & & & & $<0.01^{\ddagger}$ \\
\hline Primary school or below & $1833(69.3)$ & $762(63.4)$ & $1071(74.3)$ & - \\
\hline Junior middle school & $658(24.9)$ & $343(28.5)$ & & - \\
\hline Senior middle school & $120(4.5)$ & $73(6.1)$ & $47(3.3)$ & - \\
\hline College or above & & $24(2.0)$ & $9(0.6)$ & - \\
\hline $\begin{array}{l}\text { Family history of stroke, } \\
\mathrm{n}(\%)\end{array}$ & $474(17.9)$ & $206(17.1)$ & $268(18.6)$ & $0.33^{\ddagger}$ \\
\hline \multicolumn{5}{|l|}{ Vascular risk factors, n (\%) } \\
\hline Ischemic stroke or TIA & 468 (17.7) & $173(14.4)$ & $295(20.5)$ & $<0.01^{\ddagger}$ \\
\hline Hemorrhagic stroke & $93(3.5)$ & $36(3.0)$ & $57(4.0)$ & $0.18^{\ddagger}$ \\
\hline Hypertension & $2122(80.3)$ & 945 (78.6) & 1177 (81.6) & $0.05^{\ddagger}$ \\
\hline Diabetes mellitus & $907(34.3)$ & $341(28.4)$ & $566(39.3)$ & $<0.01^{\ddagger}$ \\
\hline Dyslipidemia & $1918(72.5)$ & $815(67.8)$ & $1103(76.5)$ & $<0.01^{\ddagger}$ \\
\hline Atrial fibrillation & $252(9.5)$ & $94(7.8)$ & $158(11.0)$ & $<0.01^{\ddagger}$ \\
\hline Smoking status, n (\%) & & & & $<0.01^{\ddagger}$ \\
\hline Never & $1736(65.7)$ & 383 (31.9) & $1353(93.8)$ & - \\
\hline Former & $192(7.3)$ & $167(13.9)$ & $25(1.7)$ & - \\
\hline Current & $716(27.1)$ & $652(54.2)$ & $64(4.4)$ & - \\
\hline Alcohol consumption, n (\%) & $248(9.4)$ & $225(18.7)$ & $23(1.6)$ & $<0.01^{\ddagger}$ \\
\hline Physical inactivity, n (\%) & $1698(64.2)$ & $757(63.0)$ & $941(65.3)$ & $0.22^{\ddagger}$ \\
\hline Overweight or obesity, n (\%) & $1647(62.3)$ & $744(61.9)$ & $903(62.6)$ & $0.69 \neq$ \\
\hline $\mathrm{BMI}, \mathrm{kg} / \mathrm{m}$ & $26.0 \pm 3.6$ & $25.9 \pm 3.3$ & $26.1 \pm 3.7$ & $0.16^{\star}$ \\
\hline Waist circumference, cm & $88.9 \pm 12.0$ & $88.9 \pm 10.0$ & $86.4 \pm 11.6$ & $<0.01^{*}$ \\
\hline Hip circumference, cm & $95.2 \pm 11.9$ & $95.9 \pm 10.5$ & $94.6 \pm 13.0$ & $0.16^{\star}$ \\
\hline Hemoglobin A1c, mmol/L & $6.8 \pm 1.8$ & $6.9 \pm 2.0$ & $6.6 \pm 1.6$ & $<0.01^{\star}$ \\
\hline $\mathrm{FBG}, \mathrm{mmol} / \mathrm{L}$ & $6.4 \pm 2.5$ & $6.3 \pm 2.2$ & $6.5 \pm 2.7$ & $0.02^{\star}$ \\
\hline Total cholesterol, mmol/L & $5.2 \pm 1.0$ & $5.1 \pm 1.0$ & $5.3 \pm 1.0$ & $<0.01^{*}$ \\
\hline $\mathrm{HDL}-\mathrm{C}, \mathrm{mmol} / \mathrm{L}$ & $1.6 \pm 0.6$ & $1.5 \pm 0.5$ & $1.7 \pm 0.6$ & $<0.01^{\star}$ \\
\hline LDL-C, mmol/L & $3.0 \pm 0.9$ & $2.9 \pm 0.9$ & $3.1 \pm 0.9$ & $<0.01^{*}$ \\
\hline Triglycerides, mmol/L & $1.8 \pm 1.9$ & $1.8 \pm 2.2$ & $1.8 \pm 1.6$ & $0.36^{\star}$ \\
\hline Homocysteine, mmol/L & $13.6 \pm 9.4$ & $15.5 \pm 11.1$ & $11.9 \pm 7.2$ & $<0.01^{\star}$ \\
\hline Total carotid plaque, n (\%) & $904(34.2)$ & $441(36.7)$ & $463(32.1)$ & $0.01^{\ddagger}$ \\
\hline $\begin{array}{l}\text { Vulnerable carotid plaque, } \\
\text { n (\%) }\end{array}$ & $425(16.1)$ & $240(20.0)$ & $185(12.8)$ & $<0.01^{\ddagger}$ \\
\hline
\end{tabular}

Data are presented as mean $\pm S D$, median (range), or number (\%).

${ }^{*}$ Student t-test. ${ }^{\ddagger} \times 2$ test.

BMI, Body mass index; FBG, fasting blood glucose; HDL-C, high density lipoprotein cholesterol; LDL-C, low density lipoprotein cholesterol.

Carotid plaques were found in 904 (34.2\%) subjects, and 425 (16.1\%) had vulnerable plaques. The total prevalence of carotid plaque was higher in men than in women $(36.7 \%$ vs. $32.1 \%$, $p=0.01)$, as well as vulnerable plaque $(20.0 \%$ vs. $12.8 \%$, $p<0.01)$.

\section{Risk Factors Associated With Carotid Plaques in High-Risk Individuals for Stroke}

Univariable analysis of risk factors associated with total carotid plaques and vulnerable plaques was presented in Table 2. Male sex was associated with both carotid plaque
TABLE 2 | Univariable analysis for the factors associated with carotid plaques in a population at high risk of stroke.

\begin{tabular}{|c|c|c|c|c|}
\hline \multirow[t]{2}{*}{ Variables* } & \multicolumn{2}{|c|}{ Total carotid plaque } & \multicolumn{2}{|c|}{ Vulnerable carotid plaque } \\
\hline & OR $(95 \% \mathrm{Cl})$ & $P$-value & OR $(95 \% \mathrm{Cl})$ & $P$-value \\
\hline Age, year & $1.07(1.06-1.08)$ & $<0.01$ & $1.05(1.04-1.06)$ & $<0.01$ \\
\hline Male & $1.23(1.04-1.44)$ & 0.01 & $1.70(1.37-2.09)$ & $<0.01$ \\
\hline \multicolumn{5}{|l|}{ Education level } \\
\hline College or above & Reference & - & Reference & - \\
\hline $\begin{array}{l}\text { Primary school or } \\
\text { below }\end{array}$ & $1.22(0.59-2.52)$ & 0.60 & $1.56(0.55-4.48)$ & 0.41 \\
\hline Junior middle school & $0.65(0.31-1.38)$ & 0.26 & $0.98(0.33-2.85)$ & 0.96 \\
\hline Senior middle school & $0.93(0.41-1.10)$ & 0.86 & $1.28(0.40-4.08)$ & 0.68 \\
\hline Family history of stroke & $0.82(0.66-1.02)$ & 0.07 & $0.77(0.57-1.02)$ & 0.07 \\
\hline \multicolumn{5}{|l|}{ Vascular risk factors } \\
\hline Ischemic stroke or TIA & $0.71(0.57-0.88)$ & $<0.01$ & $0.88(0.67-1.17)$ & 0.39 \\
\hline Hemorrhagic stroke & $1.41(0.93-2.14)$ & 0.11 & $1.09(0.63-1.88)$ & 0.76 \\
\hline Hypertension & $1.67(1.35-2.07)$ & $<0.01$ & $1.64(1.22-2.19)$ & $<0.01$ \\
\hline Dyslipidemia & $1.22(1.02-1.47)$ & 0.03 & $1.20(0.95-1.53)$ & 0.13 \\
\hline Diabetes mellitus & $1.06(0.90-1.26)$ & 0.50 & $1.09(0.88-1.36)$ & 0.42 \\
\hline Atrial fibrillation & $1.12(0.85-1.47)$ & 0.42 & $1.02(0.71-1.44)$ & 0.93 \\
\hline \multicolumn{5}{|l|}{ Smoking status } \\
\hline Never & Reference & - & Reference & - \\
\hline Former & $2.04(1.51-2.75)$ & $<0.01$ & $1.93(1.34-2.77)$ & $<0.01$ \\
\hline Current & $1.36(1.13-1.63)$ & $<0.01$ & $1.70(1.36-2.14)$ & $<0.01$ \\
\hline Alcohol consumption & $1.13(0.86-1.48)$ & 0.38 & $1.11(0.78-1.56)$ & 0.57 \\
\hline Physical inactivity & $1.00(0.84-1.18)$ & 0.96 & $0.92(0.74-1.14)$ & 0.44 \\
\hline BMI & $1.00(0.98-1.02)$ & 0.99 & $0.97(0.95-1.00)$ & 0.09 \\
\hline Waist circumference & $1.00(0.99-1.01)$ & 0.38 & $1.00(0.99-1.01)$ & 0.85 \\
\hline Hip circumference & $1.00(0.98-1.01)$ & 0.78 & $0.99(0.97-1.01)$ & 0.38 \\
\hline \multicolumn{5}{|l|}{ laboratory test } \\
\hline Hemoglobin A1c & $1.08(1.01-1.16)$ & 0.03 & $1.09(1.01-1.18)$ & 0.03 \\
\hline FBG & $1.04(1.01-1.08)$ & 0.01 & $1.05(1.01-1.09)$ & 0.01 \\
\hline Triglycerides & $1.01(0.97-1.05)$ & 0.71 & $1.05(1.01-1.11)$ & 0.03 \\
\hline TC & $1.19(1.10-1.29)$ & $<0.01$ & $1.21(1.10-1.33)$ & $<0.01$ \\
\hline HDL-C & $1.20(1.04-1.37)$ & 0.01 & $1.05(0.88-1.24)$ & 0.59 \\
\hline LDL-C & $1.22(1.11-1.34)$ & $<0.01$ & $1.26(1.12-1.42)$ & $<0.01$ \\
\hline Homocysteine & $1.01(1.00-1.02)$ & 0.06 & $1.00(0.99-1.01)$ & 0.72 \\
\hline
\end{tabular}

OR, odds ratio; $\mathrm{Cl}$, confidence intervals; $B M I$, Body mass index; $F B G$, fasting blood glucose; TC, total cholesterol; HDL-C, high density lipoprotein cholesterol; $L D L-C$, low density lipoprotein cholesterol.

(OR 1.23, 95\%CI 1.04-1.44, $p=0.01$ ) and vulnerable plaque (OR 1.70, 95\%CI 1.37-2.09, $p<0.01$ ). Besides, age, history of ischemic stroke/TIA, hypertension, dyslipidemia, former or current smoking, baseline hemoglobin A1c, FBG, TC, and LDL-C were associated with total carotid plaque (all $p<0.05)$. Meanwhile, age, hypertension, former or current smoking, baseline hemoglobin A1c, FBG, triglycerides, TC, and LDL-C were associated with vulnerable plaque (all $p<0.05)$. 


\section{The Association Between Male Sex and Carotid Plaque in High-Risk Individuals for Stroke}

As presented in Table 3, multivariate logistic regression was conducted to explore the association between male sex and total carotid plaque or vulnerable plaque. After adjusting for age, family history of stroke, and BMI (model 1 or 2), male sex was significantly associated with total carotid plaque $(p<0.01)$ and vulnerable plaque $(p<0.01)$. When vascular risk factors (including a history of ischemic stroke or TIA, hypertension, dyslipidemia, diabetes mellitus, smoking status) and lab tests (including hemoglobin A1c, FBG, triglycerides, TC, HDL-C, LDL-C) were included in the multivariate logistic regression (model 3 or 4), male sex was no longer an independent risk factor for carotid plaque, however, the male was still an independent risk factor for vulnerable plaque (adjusted OR 1.70, 95\%CI 1.10-2.62, $p=0.02$, in model 4) than female.

\section{Stratified Analyses and Interaction Test of the Association Between Male Sex and Vulnerable Plaque}

To further explore the association between male sex and vulnerable carotid plaques, stratified analyses and interaction tests were employed. In Figure 2, we found that the association between male sex and vulnerable carotid plaque did not change by age, family history of stroke, histories of chronic disease (ischemic stroke or TIA, hypertension, dyslipidemia, diabetes mellitus), smoking status, drinking status, physical activity and BMI (all $p$ for interaction $>0.05$ ). Male individuals tended to have a stronger association with vulnerable carotid plaque compared with the female, as shown in Table 3.

\section{Gender Differences in the Prevalence of Carotid Plaque and Vulnerable Plaque After PSM}

After PSM, we identified two subgroups of 928 participants (including 464 men and 464 women) at high risk of stroke that were balanced for all characteristics. Relative multivariate imbalance in terms of the L1 measure was smaller ( 0.468 vs. 0.733 ) and no covariate had standardized mean differences $>0.1$ after PSM. As shown in Table 4, there was no significant difference in the characteristics between different gender groups in the matched dataset (all $p>0.05$ ). After PSM, men no longer had more carotid plaque (33.2\% vs. $31.9 \%, p=0.67)$, however, vulnerable plaques were still more prevalent in male individuals (17.0\% vs. $12.1 \%, p=0.03)$.

\section{DISCUSSION}

Atherosclerosis in the carotid artery can lead to plaque vulnerability, which is one of the main causes of ischemic stroke (Howard et al., 2015; Pelisek et al., 2012). Our present study have identified a high prevalence of total
TABLE 3 | Multiple logistic regression analysis for the association between male sex and carotid plaque in a population at high risk of stroke.

\begin{tabular}{|c|c|c|c|c|}
\hline & \multicolumn{2}{|c|}{ Total carotid plaque } & \multicolumn{2}{|c|}{ Vulnerable carotid plaque } \\
\hline & OR $(95 \% \mathrm{Cl})$ & $P$-value & OR $(95 \% \mathrm{Cl})$ & $P$-value \\
\hline Unadjusted & $1.23(1.04-1.44)$ & 0.01 & 1.70 (1.37-2.09) & $<0.01$ \\
\hline Model 1 & $1.33(1.12-1.58)$ & $<0.01$ & $1.79(1.45-2.22)$ & $<0.01$ \\
\hline Model 2 & $1.35(1.14-1.60)$ & $<0.01$ & $1.79(1.45-2.22)$ & $<0.01$ \\
\hline Model 3 & $1.03(0.82-1.29)$ & 0.82 & $1.49(1.13-1.97)$ & $<0.01$ \\
\hline Model 4 & 1.30 (0.89-1.89) & 0.17 & $1.70(1.10-2.62)$ & 0.02 \\
\hline
\end{tabular}

Model 1 was adjusted for age and family history of stroke.

Model 2 was adjusted for variables in model $1+$ BMI.

Model 3 was adjusted for variables in model $1+B M I+$ vascular risk factors (history of ischemic stroke or TIA, hypertension, dyslipidemia, diabetes mellitus, smoking status).

Model 4 was adjusted for variables in model $1+B M I+$ vascular risk factors + laboratory test (Hemoglobin A1C, FBG, Triglycerides, TC, HDL-C, LDL-C). OR, odds ratio; Cl, confidence intervals; BMI, Body mass index; FBG, fasting blood glucose; TC, total cholesterol; HDL-C, high density lipoprotein cholesterol; $L D L-C$, low density lipoprotein cholesterol.

carotid plaque (34.2\%) and vulnerable carotid plaque (16.1\%) among high-risk participants for stroke in southwestern China and demonstrated that male individuals have a higher risk of vulnerable plaques than the female (adjusted OR 1.70, 95\% CI 1.10-2.62), even after propensity score -matched. Moreover, stratified analyses and interaction tests showed that the stronger association between male sex and vulnerable plaque did not change by age, family history of stroke, histories of chronic disease (ischemic stroke or TIA, hypertension, dyslipidemia, diabetes mellitus), smoking status, drinking, physical activity, and BMI, suggesting that male is associated with a higher risk of vulnerable plaque independent of classical vascular risk factors.

It has been demonstrated that age, hypertension, diabetes, high low-density lipoprotein cholesterol levels, and current smoking are traditional cardiovascular risk factors related to the prevalence of carotid plaques (Sturlaugsdottir et al., 2016; Bian et al., 2018; Noflatscher et al., 2019; Santos-Neto et al., 2021). However, there is scarce information regarding the gender differences in the prevalence of carotid plaques in participants at high risk of stroke, especially vulnerable plaque. It is known that IPH is one of the major characteristics of vulnerable plaque, several researchers have investigated the association between sex and IPH in the carotid artery (Hellings et al., 2007; Ota et al., 2010; Vrijenhoek et al., 2013). Observational research based on histological analysis of CEA specimens found that female individuals tend to have a more stable, less inflammatory carotid plaques compared with the male, independent of clinical manifestation and cardiovascular risk factors (Hellings et al., 2007). Similarly, a cohort study conducted in patients who had undergone CEA suggested that carotid plaques obtained from male individuals had a higher prevalence of IPH compared with the female (Vrijenhoek et al., 2013). Another study enrolled patients with asymptomatic moderate or severe carotid stenosis, and suggested that men had more highrisk plaques compared with women after justing for potential 


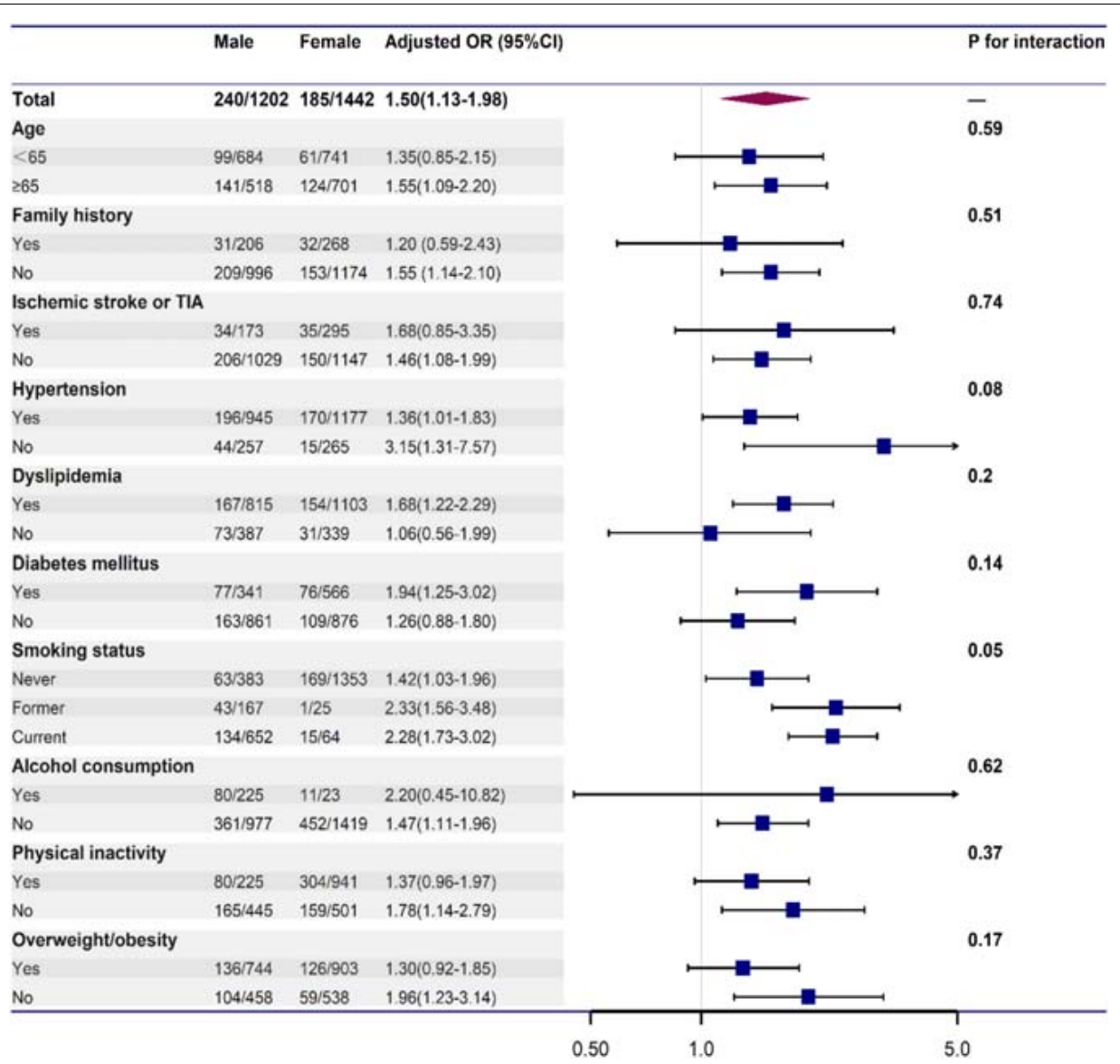

FIGURE 2 | Stratified logistic regression analysis to identify variables that modify the association between male and vulnerable carotid plaque. Each stratification was adjusted for age, family history of stroke, histories of chronic disease (ischemic stroke or TIA, hypertension, dyslipidemia, diabetes mellitus), smoking status, regular alcohol consumption, physical inactivity, and BMl, except for the stratification factor itself.

confounders (Ota et al., 2010). Our study demonstrated that male individuals had a higher risk of vulnerable carotid plaques than the females, which is similar to the results of previous studies (Hellings et al., 2007; Ota et al., 2010; Vrijenhoek et al., 2013). The difference between our study and previous studies is that the current study was a population-based study conducted in high-risk individuals for stroke, which is different from previous studies that mainly focused on patients with moderate or severe carotid stenosis, even posttreatment of CEA.

The underlying pathophysiologic mechanisms that explain these gender differences of the prevalence of vulnerable carotid plaque are poorly understood. There are several possible reasons for this. First, although men do not experience a rapid decline in endogenous sex hormone production, an age-related decrease in the levels of endogenous sex hormone especially testosterone might have an important effect on the progression of atherosclerosis. It has been demonstrated that low levels of free testosterone are associated with the progression of carotid atherosclerosis in elderly men independently of classical vascular risk factors (Muller et al., 2004; Svartberg et al., 2006; Soisson et al., 2012). Second, there are differences in the protective effect of estrogen on atherosclerosis between the two genders (Yahagi et al., 2015). Estrogen might play a direct effect on matrix metalloproteinase production contributing to the attenuation of atherosclerotic disease in females (Yahagi et al., 2015). A recently published observational study found that men have more agespecific carotid IPH in magnetic resonance imaging compared with women. However, among post-menopausal women, the risk of carotid IPH becomes closer to that of men with increasing age (Singh et al., 2017). It has been found that men with the common genetic variation in estrogen receptor alpha have three times higher risk of myocardial infarction as compared to those without variant (Shearman et al., 2003), which indicates that genetic factors might play an essential role in the gender differences of atherosclerosis. A previous study found that only $19.5 \%$ of the carotid plaque burden could be explained by traditional and less traditional vascular risk factors, also suggesting that genetic and environmental factors might play a major role in the determination of atherosclerosis (Kuo et al., 2012). Until recently, variation in genes related to inflammation, endothelial function, and lipid metabolism are thought to be linked to carotid plaque burden (Gardener et al., 2011; Wang et al., 2011; Stroke Prevention Project Committee, 2018). Whether there is a gender-specific association between variations in genes related to inflammation, endothelial function, and lipid metabolism and plaque vulnerability has not been adequately studied. 
TABLE 4 | Gender differences in the characteristics of individuals at high risk of stroke and the prevalence of carotid plaque after PSM.

\begin{tabular}{|c|c|c|c|c|}
\hline Variables & $\begin{array}{c}\text { Total } \\
(n=928)\end{array}$ & $\begin{array}{c}\text { Male } \\
(n=464)\end{array}$ & $\begin{array}{l}\text { Female } \\
(n=464)\end{array}$ & $P$-value \\
\hline Age, year, mean $\pm S D$ & $63.1 \pm 10.0$ & $63.2 \pm 10.4$ & $63.0 \pm 9.7$ & $0.77^{\star}$ \\
\hline Education, n (\%) & & & & $0.215^{\ddagger}$ \\
\hline $\begin{array}{l}\text { Primary school or } \\
\text { below }\end{array}$ & $620(66.8)$ & 297 (64.0) & $323(69.6)$ & - \\
\hline Junior middle school & $256(27.6)$ & 136 (29.3) & $120(25.9)$ & - \\
\hline Senior middle school & $42(4.5)$ & $26(5.6)$ & $16(3.5)$ & - \\
\hline College or above & $10(1.1)$ & $5(1.1)$ & $5(1.1)$ & - \\
\hline $\begin{array}{l}\text { Family history of stroke, } \\
\mathrm{n}(\%)\end{array}$ & 179 (19.3) & 85 (18.3) & $94(20.3)$ & $0.45^{\ddagger}$ \\
\hline \multicolumn{5}{|l|}{$\begin{array}{l}\text { Vascular risk factors, } \\
\mathrm{n}(\%)\end{array}$} \\
\hline Ischemic stroke & $151(16.3)$ & $72(15.5)$ & $79(17.0)$ & $0.53^{\ddagger}$ \\
\hline Hemorrhagic stroke & $33(3.6)$ & $19(4.1)$ & $14(3.0)$ & $0.38^{\ddagger}$ \\
\hline Hypertension & $763(82.2)$ & $383(82.5)$ & $380(81.9)$ & $0.80^{\ddagger}$ \\
\hline Diabetes mellitus & $328(35.3)$ & $158(34.1)$ & $170(36.6)$ & $0.41^{\ddagger}$ \\
\hline Dyslipidemia & $651(70.2)$ & $332(71.6)$ & $319(68.8)$ & $0.35^{\ddagger}$ \\
\hline Atrial fibrillation & $89(9.6)$ & $47(10.1)$ & $42(9.1)$ & $0.58^{\ddagger}$ \\
\hline Smoking status, n (\%) & & & & $0.28^{\ddagger}$ \\
\hline Never & $750(80.8)$ & 375 (80.8) & 375 (80.8) & \\
\hline Former & $41(4.4)$ & $16(3.5)$ & $25(5.4)$ & \\
\hline Current & $137(14.8)$ & $73(15.7)$ & $64(13.8)$ & \\
\hline $\begin{array}{l}\text { Alcohol consumption, } \\
\mathrm{n}(\%)\end{array}$ & $51(5.5)$ & $29(6.3)$ & $22(4.7)$ & $0.31^{\ddagger}$ \\
\hline Physical inactivity, n (\%) & 587 (63.3) & 285 (61.4) & $302(65.1)$ & $0.25^{\ddagger}$ \\
\hline $\begin{array}{l}\text { Overweight or obesity, } \\
\text { n (\%) }\end{array}$ & $595(64.1)$ & 301 (64.9) & $294(63.4)$ & $0.63^{\ddagger}$ \\
\hline $\mathrm{BMl}, \mathrm{kg} / \mathrm{m} 2$ & $26.5 \pm 3.5$ & $26.2 \pm 3.3$ & $26.3 \pm 3.8$ & $0.53^{\star}$ \\
\hline $\begin{array}{l}\text { Waist circumference, } \\
\mathrm{cm}\end{array}$ & $89.2 \pm 8.9$ & $87.0 \pm 11.2$ & $86.6 \pm 10.6$ & $0.61^{\star}$ \\
\hline Hip circumference, cm & $95.2 \pm 11.0$ & $95.9 \pm 9.8$ & $94.5 \pm 12.2$ & $0.34^{\star}$ \\
\hline FBG, mmol/L & $6.6 \pm 2.8$ & $6.5 \pm 2.7$ & $6.7 \pm 2.9$ & $0.28^{*}$ \\
\hline $\begin{array}{l}\text { Total cholesterol, } \\
\mathrm{mmol} / \mathrm{L}\end{array}$ & $5.2 \pm 1.1$ & $5.2 \pm 1.1$ & $5.3 \pm 1.1$ & $0.30^{\star}$ \\
\hline HDL-C, mmol/L & $1.7 \pm 0.7$ & $1.7 \pm 0.7$ & $1.7 \pm 0.7$ & $0.63^{\star}$ \\
\hline LDL-C, mmol/L & $3.0 \pm 0.8$ & $2.9 \pm 0.9$ & $3.0 \pm 0.8$ & $0.80^{\star}$ \\
\hline Triglycerides, mmol/L & $1.7 \pm 1.6$ & $1.7 \pm 1.6$ & $1.7 \pm 1.8$ & $0.90^{\star}$ \\
\hline Homocysteine, mmol/L & $7.8 \pm 1.3$ & $7.8 \pm 1.2$ & $7.8 \pm 1.3$ & $0.93^{*}$ \\
\hline $\begin{array}{l}\text { Total carotid plaque, } \\
\mathrm{n}(\%)\end{array}$ & $302(32.5)$ & 154 (33.2) & $148(31.9)$ & $0.67^{\ddagger}$ \\
\hline $\begin{array}{l}\text { Carotid vulnerable } \\
\text { plaque, n (\%) }\end{array}$ & $135(14.6)$ & $79(17.0)$ & $56(12.1)$ & $0.03^{\ddagger}$ \\
\hline
\end{tabular}

Data are presented as mean $\pm S D$, median (range), or number (\%).

${ }^{\star}$ Student $t$-test. ${ }^{\ddagger} \chi^{2}$-test.

BMI, Body mass index; FBG, fasting blood glucose; HDL-C, high density lipoprotein cholesterol; LDL-C, low density lipoprotein cholesterol.

\section{Limitations}

The results of the present study should be interpreted with caution given its limitations. First, although a standardized structured face-to-face questionnaire was used by experienced surveyors to collect data including demographic characteristics, behavior factors, family history of stroke, history of stroke and chronic disease, and physical examination, the application of the self-reported questionnaire might also be associated with recall bias and make the answers unreliable. Second, even though we conduct a multicenter population-based study with a large number of subjects recruited and the large number of variables collected, we only screened residents ages $\geq 40$ years and we did not compare the gender differences of carotid plaque in residents who were not identified as the high-risk individuals for stroke, therefore, our results might not represent the whole population. Third, carotid plaque and plaque vulnerability were evaluated by carotid ultrasound but not high-resolution magnetic resonance imaging, which could provide more information including plaque composition and morphology. Besides, the data collection was done many years ago and this is unlikely to provide an updated picture of the situation. Furthermore, we did not explore the effect of antiplatelet drugs or statins on plaque vulnerability in our study due to a lack of data. Finally, limited to the study protocol of the CNSSS program, we could not provide information related to inflammatory markers such as the level of C-reactive protein or other acute-phase protein, and further studies are needed to explore this issue.

\section{CONCLUSION}

Despite the above limitations, this multicenter, cross-sectional study provides clear evidence that male individuals had a higher risk of vulnerable carotid plaque independent of classical vascular risk factors, genetic factors might play a major role in the gender differences in the progression of atherosclerosis. Whether there is a gender-specific association between variations in genes involved in inflammation, endothelial function, and lipid metabolism and plaque vulnerability needs to be further studied.

\section{DATA AVAILABILITY STATEMENT}

The raw data supporting the conclusions of this article will be made available by the corresponding author on reasonable request.

\section{ETHICS STATEMENT}

Our study protocol was approved by the ethics committee of three participating hospitals (the People's Hospital of Deyang City, the Affiliated Hospital of Southwest Medical University, and the Suining Central Hospital). Informed consents were obtained from all participants during recruitment. The patients/participants provided their written informed consent to participate in this study.

\section{AUTHOR CONTRIBUTIONS}

JL and LG collected, analyzed, and interpreted the data, as well as drafted the manuscript. PZ, YL, 
and JZ participated in study conception and design, data interpretation, and revised the manuscript. XY and CW contributed substantially to study design and supervision, data interpretation, and manuscript writing. All authors critically revised the manuscript for important intellectual content and approved the final manuscript.

\section{REFERENCES}

Bian, L., Xia, L., Wang, Y., Jiang, J., Zhang, Y., Li, D., et al. (2018). Risk factors of subclinical atherosclerosis and plaque burden in high risk individuals: results from a community-based study. Front. Physiol. 9:739. doi: 10.3389/fphys.2018. 00739

Gardener, H., Beecham, A., Cabral, D., Yanuck, D., Slifer, S., Wang, L., et al. (2011). Carotid plaque and candidate genes related to inflammation and endothelial function in Hispanics from northern Manhattan. Stroke 42, 889-896. doi: 10.1161/strokeaha.110.591065

GBD 2016 Causes of Death Collaborators (2017). Global, regional, and national age-sex specific mortality for 264 causes of death, 1980-2016: a systematic analysis for the Global Burden of disease study 2016. Lancet 390, 1151-1210.

Hellings, W. E., Pasterkamp, G., Verhoeven, B. A. N., De Kleijn, D. P., De Vries, J. P., Seldenrijk, K. A., et al. (2007). Gender-associated differences in plaque phenotype of patients undergoing carotid endarterectomy. J. Vasc. Surg. 45, 289-296. doi: 10.1016/j.jvs.2006.09.051

Ho, S. S. Y. (2016). Current status of carotid ultrasound in atherosclerosis. Quant. Imaging Med. Surg. 6, 285-296. doi: 10.21037/qims.2016.05.03

Howard, D. P. J., Van Lammeren, G. W., Rothwell, P. M., Redgrave, J. N., Moll, F. L., de Vries, J. P., et al. (2015). Symptomatic carotid atherosclerotic disease: correlations between plaque composition and ipsilateral stroke risk. Stroke 46, 182-189. doi: 10.1161/strokeaha.114.007221

Kuo, F., Gardener, H., Dong, C., Cabral, D., Della-Morte, D., Blanton, S. H., et al. (2012). Traditional cardiovascular risk factors explain the minority of the variability in carotid plaque. Stroke 43, 1755-1760. doi: 10.1161/strokeaha.112. 651059

Li, J., Wang, L., Chao, B., and Liu, Y. (2015). Prevalence of stroke in China: an epidemiological study based on the national stroke screening survey. Lancet 386:S49.

Li, J., Zhang, P., Liu, Y., Wu, S., Yi, X., Zhang, S., et al. (2020a). Early amantadine treatment reduces the risk of death in patients with large hemisphere infarctions. BMC Neurol. doi: 10.1038/s41598-020-70172-1

Li, J., Zhang, P., Wu, S., Yuan, R., Liu, J., Tao, W., et al. (2020b). Impaired consciousness at stroke onset in large hemisphere infarction: incidence, risk factors and outcome. Sci. Rep. 10:13170.

Lloyd-Jones, D., Adams, R. J., Brown, T. M., Carnethon, M., Dai, S., De Simone, G., et al. (2010). Heart disease and stroke statistics-2010 update: a report from the American Heart Association. Circulation 121, e46-e215.

Mangge, H., and Almer, G. (2019). Immune-mediated inflammation in vulnerable atherosclerotic plaques. Molecules 24:3072. doi: 10.3390/molecules 24173072

Mathiesen, E. B., Bønaa, K. H., and Joakimsen, O. (2001). Low levels of highdensity lipoprotein cholesterol are associated with echolucent carotid artery plaques: the Tromsø study. Stroke 32, 1960-1965. doi: 10.1161/hs0901.09 5639

Muller, M., Van Den Beld, A. W., Bots, M. L., Grobbee, D. E., Lamberts, S. W., and van der Schouw, Y. T. (2004). Endogenous sex hormones and progression of carotid atherosclerosis in elderly men. Circulation 109, 2074-2079. doi: 10.1161/01.cir.0000125854.51637.06

Nezu, T., and Hosomi, N. (2020). Usefulness of carotid ultrasonography for risk stratification of cerebral and cardiovascular disease. J. Atheroscler. Thromb. 27, 1023-1035. doi: 10.5551/jat.rv17044

Noflatscher, M., Schreinlechner, M., Sommer, P., Kerschbaum, J., Berggren, K., Theurl, M., et al. (2019). Influence of traditional cardiovascular risk factors on carotid and femoral atherosclerotic plaque volume as measured by threedimensional ultrasound. J. Clin. Med. 8:32. doi: 10.3390/jcm8010032

Ota, H., Reeves, M. J., Zhu, D. C., Majid, A., Collar, A., Yuan, C., et al. (2010). Sex differences in patients with asymptomatic carotid atherosclerotic plaque: in vivo

\section{FUNDING}

This research was funded by Universal Application Program, Health and Family Planning Commission of Sichuan Province in China (Nos. 17PJ084 and 16ZD046), and Applied Basic Research Program, Science and Technology Department of Sichuan Province in China (No. 2018JY0389).

3.0-T magnetic resonance study. Stroke 41, 1630-1635. doi: 10.1161/strokeaha. 110.581306

Parish, S., Arnold, M., Clarke, R., Du, H., Wan, E., Kurmi, O., et al. (2019). Assessment of the role of carotid atherosclerosis in the association between major cardiovascular risk factors and ischemic stroke subtypes. JAMA Netw. Open 2:e194873. doi: 10.1001/jamanetworkopen.2019.4873

Park, T. H. (2016). Evaluation of carotid plaque using ultrasound imaging. J. Cardiovasc. Ultrasound 24, 91-95. doi: 10.4250/jcu.2016.24.2.91

Pelisek, J., Eckstein, H. H., and Zernecke, A. (2012). Pathophysiological mechanisms of carotid plaque vulnerability: impact on ischemic stroke. Arch. Immunol. Ther. Exp. 60, 431-442. doi: 10.1007/s00005-012-0192-z

Prasad, K. (2015). Pathophysiology and medical treatment of carotid artery stenosis. Int. J. Angiol. 24, 158-172. doi: 10.1055/s-0035-1554911

Puig, N., Jiménez-Xarrié, E., Camps-Renom, P., and Benitez, S. (2020). Search for reliable circulating biomarkers to predict carotid plaque vulnerability. Int. J. Mol. Sci. 21:8236. doi: 10.3390/ijms21218236

Rundek, T., Arif, H., Boden-Albala, B., Elkind, M. S., Paik, M. C., and Sacco, R. L. (2008). Carotid plaque, a subclinical precursor of vascular events: the Northern Manhattan study. Neurology. 70, 1200-1207. doi: 10.1212/01.wnl.0000303969. 63165.34

Santos-Neto, P. J., Sena-Santos, E. H., Meireles, D. P., Bittencourt, M. S., Santos, I. S., Bensenor, I. M., et al. (2021). Association of carotid plaques and common carotid intima-media thickness with modifiable cardiovascular risk factors. J. Stroke Cerebrovasc. Dis. 30:105671. doi: 10.1016/j.jstrokecerebrovasdis.2021. 105671

Shearman, A. M., Cupples, L. A., Demissie, S., Peter, I., Schmid, C. H., Karas, R. H., et al. (2003). Association between estrogen receptor $\alpha$ gene variation and cardiovascular disease. JAMA 290, 2263-2270. doi: 10.1001/jama.290.17. 2263

Sillesen, H., Sartori, S., Sandholt, B., Baber, U., Mehran, R., and Fuster, V. (2018). Carotid plaque thickness and carotid plaque burden predict future cardiovascular events in asymptomatic adult Americans. Eur. Heart J. Cardiovasc. Imaging 19, 1042-1050. doi: 10.1093/ehjci/jex239

Singh, N., Moody, A. R., Zhang, B., Kaminski, I., Kapur, K., Chiu, S., et al. (2017). Age-specific sex differences in magnetic resonance imaging-depicted carotid intraplaque hemorrhage. Stroke 48, 2129-2135. doi: 10.1161/strokeaha.117. 017877

Soisson, V., Brailly-Tabard, S., Empana, J. P., Féart, C., Ryan, J., Bertrand, M., et al. (2012). Low plasma testosterone and elevated carotid intimamedia thickness: importance of low-grade inflammation in elderly men. Atherosclerosis 223, 244-249. doi: 10.1016/j.atherosclerosis.2012. 05.009

Stroke Prevention Project Committee (2018). Program of Stroke Screening and Intervention for High-risk Population. Available online at: https://pro.chinasdc. cn (accessed November 30, 2018).

Sturlaugsdottir, R., Aspelund, T., Bjornsdottir, G., Sigurdsson, S., Thorsson, B., Eiriksdottir, G., et al. (2016). Prevalence and determinants of carotid plaque in the cross-sectional REFINE-Reykjavik study. BMJ Open 6:e012457. doi: 10. 1136/bmjopen-2016-012457

Svartberg, J., Von Mühlen, D., Mathiesen, E., Joakimsen, O., Bønaa, K. H., and Stensland-Bugge, E. (2006). Low testosterone levels are associated with carotid atherosclerosis in men. J. Int. Med. 259, 576-582. doi: 10.1111/j.1365-2796. 2006.01637.x

Vrijenhoek, J. E. P., Den Ruijter, H. M., De Borst, G. J., de Kleijn, D. P., De Vries, J. P., Bots, M. L., et al. (2013). Sex is associated with the presence of atherosclerotic plaque hemorrhage and modifies the relation between plaque hemorrhage and cardiovascular outcome. Stroke 44, 3318-3323. doi: 10.1161/ strokeaha.113.002633 
Wang, L., Yanuck, D., Beecham, A., Gardener, H., Slifer, S., Blanton, S. H., et al. (2011). A candidate gene study revealed sex-specific association between the OLR1 gene and carotid plaque. Stroke 42, 588-592. doi: 10.1161/strokeaha.110. 596841

Wang, W., Jiang, B., Sun, H., Ru, X., Sun, D., Wang, L., et al. (2017). Prevalence, incidence, and mortality of stroke in China: results from a nationwide population-based survey of 480687 adults. Circulation 135, 759-771. doi: 10.1161/circulationaha.116.025250

Wijeratne, T., Menon, R., Sales, C., Karimi, L., and Crewther, S. (2020). Carotid artery stenosis and inflammatory biomarkers: the role of inflammation-induced immunological responses affecting the vascular systems. Ann. Trans. Med. 8:1276. doi: $10.21037 / \mathrm{atm}-20-4388$

Wu, S., Wu, B., Liu, M., Chen, Z., Wang, W., Anderson, C. S., et al. (2019). Stroke in China: advances and challenges in epidemiology, prevention, and management. Lancet Neurol. 18, 394-405.

Yahagi, K., Davis, H. R., Arbustini, E., and Virmani, R. (2015). Sex differences in coronary artery disease: pathological observations. Atherosclerosis 239, 260-267. doi: 10.1016/j.atherosclerosis.2015. 01.017

Yi, X., Lin, J., Luo, H., Wang, C., and Liu, Y. (2017). Genetic variants of PTGS2, TXA2R and TXAS1 are associated with carotid plaque vulnerability, platelet activation and TXA2 levels in ischemic stroke patients. PLoS One 12:e0180704. doi: 10.1371/journal.pone. 0180704
Yi, X., Luo, H., Zhou, J., Yu, M., Chen, X., Tan, L., et al. (2020a). Prevalence of stroke and stroke related risk factors: a population based cross sectional survey in southwestern China. BMC Neurol. 20:5. doi: 10.1186/s12883-019$1592-Z$

Yi, X., Zhu, L., Sui, G., Li, J., Luo, H., Yu, M., et al. (2020b). Inflammation and endothelial function relevant genetic polymorphisms and carotid plaque in chinese population. J. Atheroscler. Thromb. 27, 978-994. doi: 10.5551/jat. 53074

Yi, X. Y., Liao, D. X., Wang, C., Cheng, W., Fu, X. Q., and Zhang, B. (2016). Cytochrome P450 genetic variants and their metabolite levels associated with plaque stability in patients with ischemic stroke. J. Atheroscler. Thromb. 23, 330-338. doi: 10.5551/jat.31120

Conflict of Interest: The authors declare that the research was conducted in the absence of any commercial or financial relationships that could be construed as a potential conflict of interest.

Copyright $\odot 2021 \mathrm{Li}, \mathrm{Gao}$, Zhang, Liu, Zhou, Yi and Wang. This is an open-access article distributed under the terms of the Creative Commons Attribution License (CC BY). The use, distribution or reproduction in other forums is permitted, provided the original author(s) and the copyright owner(s) are credited and that the original publication in this journal is cited, in accordance with accepted academic practice. No use, distribution or reproduction is permitted which does not comply with these terms. 